\title{
ARTICLE
}

Clinical Study

\section{A randomised phase II study of second-line XELIRI regimen versus irinotecan monotherapy in advanced biliary tract cancer patients progressed on gemcitabine and cisplatin}

\author{
Yi Zheng ${ }^{1}$, Xiaoxuan Tu${ }^{1}$, Peng Zhao ${ }^{1}$, Weiqin Jiang ${ }^{1}$, Lulu Liu ${ }^{1}$, Zhou Tong ${ }^{1}$, Hangyu Zhang $^{1}$, Cong Yan ${ }^{1}$, Weijia Fang ${ }^{1}$ and \\ Weilin Wang ${ }^{2}$
}

BACKGROUND: The majority of advanced biliary tract cancer (ABTC) patients will progress after gemcitabine and cisplatin (GP) doublet therapy, while the standard second-line regimen has not been established. We conducted this study to assess the efficacy and safety of second-line irinotecan and capecitabine (XELIRI) regimen vs. irinotecan monotherapy in ABTC patients progressed on GP.

METHODS: Sixty-four GP refractory ABTC patients were randomised to either irinotecan $180 \mathrm{mg} / \mathrm{m}^{2}$ on day 1 plus capecitabine $1000 \mathrm{mg} / \mathrm{m}^{2}$ twice daily on days $1-10$ of a 14-day cycle (XELIRI-arm) or single-agent irinotecan $180 \mathrm{mg} / \mathrm{m}^{2}$ on day 1 of a $14-$ day cycle (IRI-arm). Treatments were repeated until disease progression or unacceptable toxicity occurred.

RESULTS: A total of 60 patients were included in the analysis. For XELIRI and IRI-arms, respectively, the median PFS was 3.7 vs. 2.4 months, 9 -month survival rate $60.9 \%$ vs. $32.0 \%$, median OS 10.1 vs. 7.3 months, and disease control rate $63.3 \%$ vs. $50.0 \%$. The most common grade 3 or 4 toxicities were leucopaenia and neutropaenia.

CONCLUSIONS: This randomised, phase II study of irinotecan-containing regimens in good PS second-line ABTC patients showed a clear benefit of XELIRI regimen over irinotecan monotherapy in prolonging PFS, with acceptable toxicity.

British Journal of Cancer (2018) 119:291-295; https://doi.org/10.1038/s41416-018-0138-2

\section{INTRODUCTION}

Biliary tract cancer (BTC) is a rarely ${ }^{1}$ and highly fatal malignancy with a 5-year overall survival (OS) rate of only about $10 \%$ for cholangiocarcinoma and less than $5 \%$ for gallbladder cancer. ${ }^{2,3}$ Radical resection is the only potentially curative approach to early stage BTC. However, recurrence after surgical resection is common since BTC has high potential and propensity to metastasise. Moreover, at the time of diagnosis, most of the patients present with advanced stage disease which precludes the possibility of surgical resection. Therefore, palliative chemotherapy is the standard therapeutic option for advanced biliary tract cancer (ABTC).

In the past decades, several studies have demonstrated the efficacy and safety of gemcitabine in ABTC., ${ }^{4,5}$ In 2010, the combination regimen with gemcitabine plus cisplatin (GP) was shown to significantly improve the survival of patients with ABTC compared to gemcitabine alone as first-line therapy in two randomised trials (OS: 11.7 vs. 8.1 months, 11.2 vs. 7.7 months, respectively). ${ }^{6,7}$ GP doublet therapy is now the standard first-line regimen for $A B T C$.

Unfortunately, the majority of ABTC cases will eventually progress on GP doublet. Approximate $50 \%$ of these patients with good performance status (PS) undergo further treatment, ${ }^{8,9}$ but no consensus has been made for the most suitable regimen in the second-line setting. ${ }^{10-12}$ There were several studies evaluating the potential effects of irinotecan as a monotherapy or as part of a combination in ABTC, and its anti-tumour effect was encouraging with acceptable toxicity. ${ }^{13-16}$ More recently, capecitabine has shown a survival benefit over observation alone for adjuvant therapy in BTC in a phase III randomised trial, with little reported impact on quality of life. ${ }^{17}$ However, the role of irinotecan and capecitabine (XELIRI) regimen in second-line ABTC chemotherapy remains an unresolved issue.

Given the promising results from the previous studies, we conducted this study to compare the efficacy and safety of secondline XELIRI regimen to irinotecan monotherapy in good PS ABTC patients with progressive disease following GP doublet chemotherapy, as a randomised prospective phase II study. The primary objective of this study was to compare the progression-free survival (PFS) in ABTC patients who received one of these two therapies. The secondary objectives were OS, response rate (RR) and assessment of safety.

\section{MATERIALS AND METHODS}

Study design

This was a single-centre, randomised phase II study to evaluate the efficacy and safety of XELIRI regimen compared with

\footnotetext{
${ }^{1}$ Cancer Biotherapy Centre, the First Affiliated Hospital of Zhejiang University, Hangzhou 310003, China and ${ }^{2}$ Department of Hepatobiliary Pancreatic Surgery, the First Affiliated Hospital of Zhejiang University, Hangzhou 310003, China

Correspondence: Weilin Wang (wam@zju.edu.cn)

These authors contributed equally: Yi Zheng, Xiaoxuan Tu.
}

Received: 10 February 2018 Revised: 13 May 2018 Accepted: 16 May 2018

Published online: 29 June 2018 
irinotecan monotherapy in GP doublet refractory, good PS ABTC patients. Patients were randomised to either single-agent irinotecan $180 \mathrm{mg} / \mathrm{m}^{2}$ on day 1 of a 14-day cycle (IRI-arm) or irinotecan $180 \mathrm{mg} / \mathrm{m}^{2}$ on day 1 plus capecitabine $1000 \mathrm{mg} / \mathrm{m}^{2}$ twice daily on days 1-10 of a 14-day cycle (XELIRI-arm). The assigned treatment was delivered until disease progression, unacceptable toxicity or patient refusal.

\section{Eligibility criteria}

Patients were eligible for the study if they were 18 years of age or older and had a histologically confirmed diagnosis of locally advanced or metastatic biliary tract adenocarcinoma (intrahepatic or extrahepatic cholangiocarcinoma, and gallbladder carcinoma) and a radiologically confirmed progression after first-line GP doublet chemotherapy, an Eastern Cooperative Oncology Group (ECOG) PS of 0 or 1 . Other eligibility criteria were radiological measurable disease, adequate function of major organs, in particular a haemoglobin $\geq 10$ g per $100 \mathrm{ml}$, white blood cells $\geq 3000 / \mathrm{mm}^{3}$, neutrophils $\geq 1500 / \mathrm{mm}^{3}$, platelets $\geq 80,000 / \mathrm{mm}^{3}$, total bilirubin levels $\leq 2$ times the upper limit of the normal range, liver-enzyme (alanine aminotransferase (ALT)/ aspartate aminotransferase (AST)) levels $\leq 5$ times the upper limit of the normal range, renal function with levels of serum creatinine $\leq 1.5$ times the upper limit of the normal range, and a calculated glomerular filtration rate $\geq 45 \mathrm{ml} / \mathrm{min}$.

This study (ClinicalTrials.Gov ID: NCT02558959) was approved by Ethics Committee of the First Affiliated Hospital of Zhejiang University and was conducted according to the Declaration of Helsinki and guidelines on Good Clinical Practice. Written informed consent was obtained from each patient before random assignment.

Efficacy and safety assessment

All patients who received at least one dose of the study drug were included in the efficacy and safety assessment. Medical records of each patient were reviewed to collect relevant data on demographics, tumour characteristics, surgery, biliary stenting, and serum levels of carbohydrate antigen 19-9 (CA 19-9) one-day before the start of second-line treatment. Tumour response was assessed in each patient every 6 weeks by means of computed tomography or magnetic resonance imaging according to the Response Evaluation Criteria in Solid Tumour (RECIST, version 1.1). PFS was defined as the time interval between the initiation of the second-line chemotherapy and disease progression or death, whichever occurred first. Toxicities were graded according to the National Cancer Institute's CTCAE v4.0. Information on third-line chemotherapy after disease progression was also collected.

\section{Statistical analysis}

The sample size was calculated by the selection method of Simon et al., ${ }^{18}$ which is based on the previous reports and the assumption that XELIRI regimen could prolong the PFS for 1.2 months than irinotecan monotherapy. ${ }^{15,16}$ With these assumptions, 30 patients per arm were needed to appropriately select the combination therapy with a probability of $\geq 80 \%$. PFS and OS were estimated using the Kaplan-Meier method. Surviving patients without disease progression were censored at the end of follow-up (January 2018). A Cox proportional hazards model was used to calculate the hazard ratio (HR), 95\% confidence interval $(\mathrm{Cl})$ and its two-tailed $P$-value. Fisher's exact test was used to compare the patient characteristics, response and disease control rates, and toxicities between the two treatment arms. A two-sided $P$-value less than 0.05 was considered significant. All statistical analyses were performed with SPSS software (version 21.0; IBM Corporation, Armonk, NY, USA).

\section{RESULTS}

\section{Patients}

This study was carried out in the First Affiliated Hospital of Zhejiang University from September 2015 to September 2017.
Sixty-four patients were randomised to either XELIRI-arm or IRIarm. Each arm has two patients not treated because of the early deterioration of general condition before study treatment. All of the remaining 60 patients, 30 in the XELIRI-arm and 30 in the IRIarm, received at least one dose of study treatment. Efficacy and safety were evaluated for each of these 60 patients (Fig. 1). Baseline characteristics (Table 1) were well balanced between the two arms.

Treatment compliance

A total of 159 and 137 cycles were administered at the time of analysis in the XELIRI-arm and IRI-arm, respectively. At the end of the first 8 weeks, treatment compliance was similar in the two groups, with $56.7 \%$ receiving four cycles of XELIRI and $60.0 \%$ receiving four cycles of irinotecan alone; however, in the treatment period overall, more patients in the IRI-arm discontinued treatment prematurely, primarily because of disease progression. In the first 8 weeks of treatment, an average of $91 \%$ of the planned dose was delivered to patients in the XELIRIarm, as compared with $89 \%$ in the IRI-arm; however, in the second 8 weeks, the average was $85 \%$ in the XELIRI-arm as compared with $67 \%$ in the IRI-arm.

\section{Efficacy}

A total of 60 patients were evaluable for tumour response according to the protocol, 30 in the XELIRI-arm and 30 in the IRIarm. In total, two complete responses (CR) were observed in the XELIRI-arm, but no CR was observed in the IRI-arm (6.7\% vs. $0 \%)$. Besides, both arms had 2 patients achieved partial response (PR) (6.7\% vs. $6.7 \%)$. The RR was higher in XELIRI-arm (13.7\% vs. $6.7 \%, P$ $=0.389$ ). In addition, 15 patients had stable disease (SD) in the XELIRI-arm, while 13 patients in the IRI-arm had SD (50.0\% vs. 43.3\%). The disease control rate (CR + PR + SD) was $63.3 \%$ vs. $50.0 \%$ in favour of the combination therapy $(P=0.297)$. The median PFS (3.7 months vs. 2.4 months, $P=0.036$ ) and 9-month survival rate $(60.9 \%$ vs. $32.0 \%, P=0.045)$ were better for the XELIRI-arm compared to IRI-arm. However, the prolonged OS (10.1 months vs. 7.3 months, $P=0.107$ ) was not statistically significant (Fig. 2, Table 2).

For prognostic factor analysis, the survival of ABTC patients with CA $19-9 \geq 400 \mathrm{IU} / \mathrm{ml}$ was worse than that of patients with CA 19-9 $<400 \mathrm{IU} / \mathrm{ml}$. Moreover, the PFS were longer with XELIRI combination in non-gallbladder cancer patients (4.9 vs. 2.4 months), as well as in patients with CA $19-9<400 \mathrm{IU} / \mathrm{ml}$ (4.3 vs. 2.8 months), as shown in Table 3.

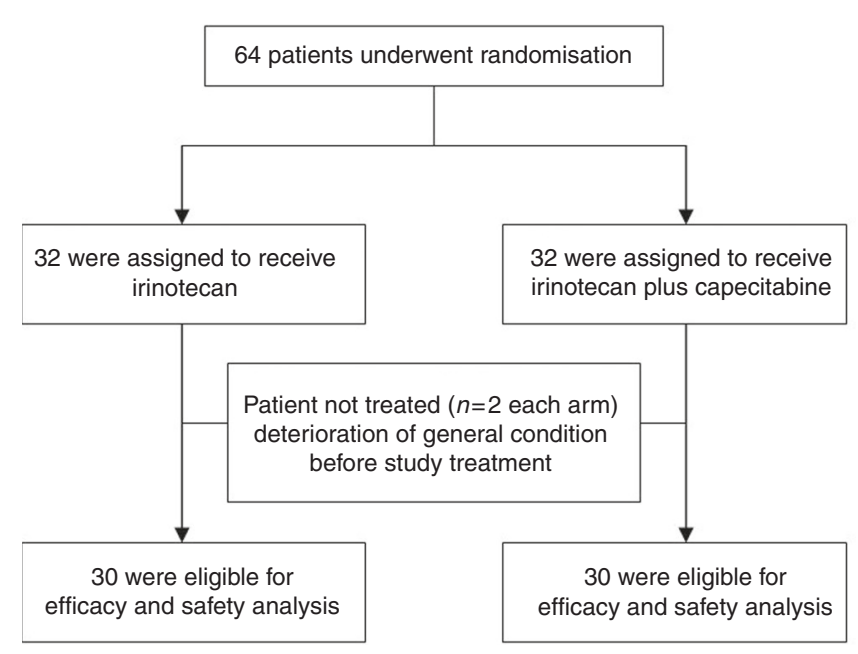

Fig. 1 Patient enrolment, randomisation and treatment 
Table 1. Baseline characteristics of the patients

\begin{tabular}{|c|c|c|c|}
\hline Characteristic & $\begin{array}{l}\text { XELIRI }(n=30) \\
n(\%)\end{array}$ & $\begin{array}{l}\text { IRI }(n=30) \\
n(\%)\end{array}$ & $P$-value \\
\hline \multicolumn{4}{|l|}{ Gender } \\
\hline Male & $16(53.3)$ & $19(63.3)$ & \\
\hline Female & $14(46.7)$ & $11(36.7)$ & 0.432 \\
\hline \multicolumn{4}{|l|}{ Age (year) } \\
\hline Median & 54 & 55 & $0.979^{a}$ \\
\hline Range & $26-70$ & $40-68$ & \\
\hline \multicolumn{4}{|l|}{ ECOG PS } \\
\hline 0 & $24(80.0)$ & $25(83.3)$ & \\
\hline 1 & $6(20.0)$ & $5(16.7)$ & 0.739 \\
\hline \multicolumn{4}{|l|}{ Extent of disease } \\
\hline Locally advanced & $14(46.7)$ & $16(53.3)$ & \\
\hline Metastatic & $16(53.3)$ & $14(46.7)$ & 0.606 \\
\hline \multicolumn{4}{|l|}{ Primary tumour site } \\
\hline Intrahepatic bile duct & $20(66.7)$ & $21(70.0)$ & \\
\hline Extrahepatic bile duct & $3(10.0)$ & $4(13.3)$ & \\
\hline Gallbladder & $7(23.3)$ & $5(16.7)$ & 0.779 \\
\hline \multicolumn{4}{|l|}{ Biliary stenting } \\
\hline Yes & $3(10.0)$ & $2(6.7)$ & \\
\hline No & $27(90.0)$ & $28(93.3)$ & 0.64 \\
\hline \multicolumn{4}{|l|}{ Previous surgery } \\
\hline Yes & $24(80.0)$ & $26(86.7)$ & \\
\hline No & $6(20.0)$ & $4(13.3)$ & 0.488 \\
\hline \multicolumn{4}{|l|}{ First-line PFS (months) } \\
\hline Median & 6.7 & 6.9 & $0.527^{\mathrm{a}}$ \\
\hline
\end{tabular}

Table 2. Summary of efficacy: overall response and survival

\begin{tabular}{|c|c|c|c|}
\hline Parameter & $\begin{array}{l}\text { XELIRI }(n=30) \\
n(\%)\end{array}$ & $\begin{array}{l}\text { IRI }(n=30) \\
n(\%)\end{array}$ & $P$-value \\
\hline \multicolumn{4}{|l|}{ Overall response rate } \\
\hline $\begin{array}{l}\text { Complete response } \\
\text { (CR) }\end{array}$ & $2(6.7)$ & $0(0.0)$ & \\
\hline Partial response (PR) & $2(6.7)$ & $2(6.7)$ & \\
\hline Stable disease (SD) & $15(50.0)$ & $13(43.3)$ & \\
\hline $\begin{array}{l}\text { Progressive disease } \\
\text { (PD) }\end{array}$ & $11(36.7)$ & $15(50.0)$ & \\
\hline Response rate (RR) & $13.3 \%$ & $6.7 \%$ & 0.389 \\
\hline $\begin{array}{l}\text { Disease control rate } \\
\text { (DCR) }\end{array}$ & $63.3 \%$ & $50.0 \%$ & 0.297 \\
\hline \multicolumn{4}{|c|}{ Progression-free survival (PFS) } \\
\hline Median PFS $(95 \% \mathrm{Cl})$ & $\begin{array}{l}3.7 \text { months }(0.3 \text {, } \\
7.1)\end{array}$ & $\begin{array}{l}2.4 \text { months (2.0, } \\
2.8)\end{array}$ & $0.036^{*}$ \\
\hline $\begin{array}{l}\text { Hazard ratio }(95 \% \\
\mathrm{Cl})\end{array}$ & \multicolumn{3}{|c|}{0.54 (95\% Cl: $0.30,0.98)$} \\
\hline \multicolumn{4}{|l|}{ Overall survival (OS) } \\
\hline $\begin{array}{l}9 \text { months survival } \\
\text { rate }\end{array}$ & $60.9 \%$ & $32.0 \%$ & $0.045^{*}$ \\
\hline $\begin{array}{l}\text { Hazard ratio }(95 \% \\
\mathrm{Cl})\end{array}$ & \multicolumn{3}{|c|}{0.30 (95\% Cl: 0.09, 0.99) } \\
\hline Median OS $(95 \% \mathrm{Cl})$ & $\begin{array}{l}10.1 \text { months (7.4, } \\
12.8)\end{array}$ & $\begin{array}{l}7.3 \text { months (6.1, } \\
8.5)\end{array}$ & 0.107 \\
\hline $\begin{array}{l}\text { Hazard ratio }(95 \% \\
\mathrm{Cl})\end{array}$ & \multicolumn{3}{|c|}{0.63 (95\% Cl: $0.35,1.12)$} \\
\hline
\end{tabular}
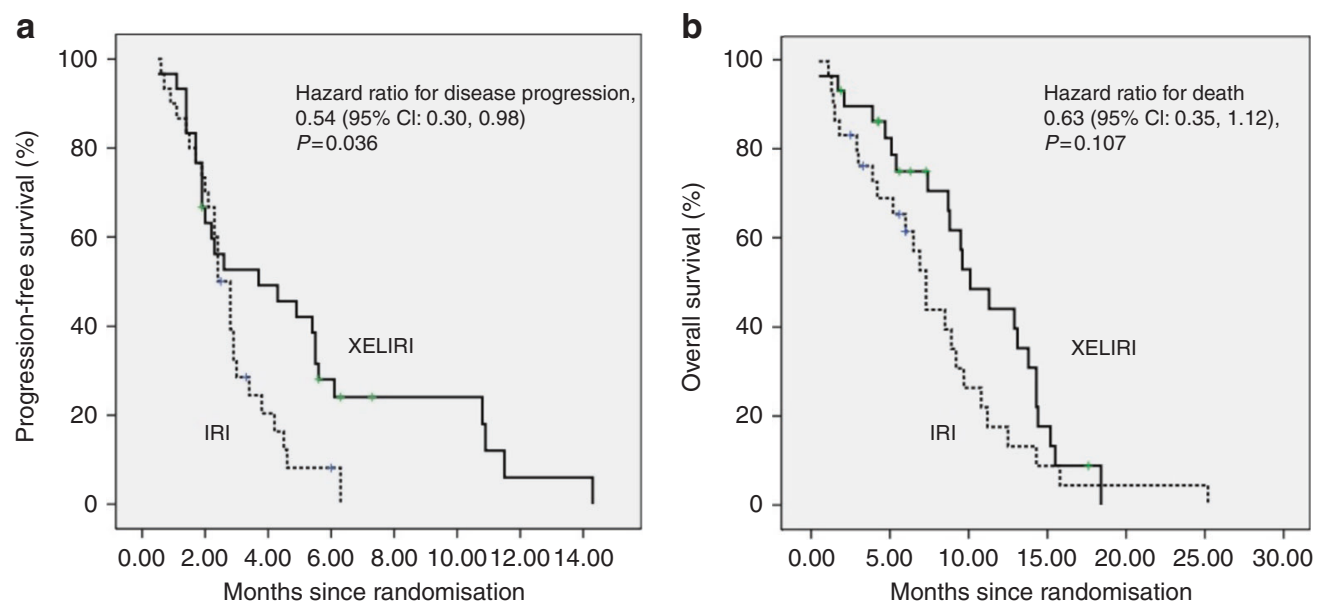

Fig. 2 Kaplan-Meier curve of progression-free survival and overall survival. a Progression-free survival. b Overall survival. XELIRI irinotecan and capecitabine combination, IRI irinotecan monotherapy, $\mathrm{Cl}$ confidence interval

\section{Safety}

All adverse events recorded in this study were predictable and manageable based on the safety profile of irinotecan and capecitabine. Table 4 describes the main adverse events which occurred during the study. Generally, toxicities were mild: the most common events $(\geq 50 \%)$ were leucopaenia, neutropaenia, nausea, anaemia and hand-foot syndrome in the XELIRI-arm, and leucopaenia, neutropaenia, nausea, thrombocytopaenia and anaemia in the IRI-arm. The incidence of hand-foot syndrome and stomatitis was higher in the XELIRI-arm, which is common in capecitabine-containing regimens. No treatment related death occurred. Most of the patients recovered from the adverse events by supportive care or reducing the dose intensity.

Post-study chemotherapy

Among the patients who went on to receive third-line therapy, 13 patients $(43.3 \%)$ in the XELIRI-arm received post-study 
Table 3. Prognostic factor analysis of progression-free survival time

\begin{tabular}{|c|c|c|c|}
\hline \multirow[t]{2}{*}{ Parameter } & \multicolumn{2}{|c|}{$\begin{array}{l}\text { Median progression-free } \\
\text { survival time (months) }(95 \% \mathrm{Cl})\end{array}$} & \multirow[t]{2}{*}{$P$-value } \\
\hline & XELIRI $(n=30)$ & $\mathrm{IRI}(n=30)$ & \\
\hline \multicolumn{4}{|l|}{ Tumour site } \\
\hline Gallbladder & $2.3(1.3,3.3)$ & $2.8(1.9,3.7)$ & 0.689 \\
\hline Non-gallbladder & $4.9(0.7,9.1)$ & $2.4(1.8,3.0)$ & $0.032^{*}$ \\
\hline \multicolumn{4}{|l|}{ CA 19-9 } \\
\hline$\geq 400 \mathrm{IU} / \mathrm{ml}$ & $2.3(0.1,5.0)$ & $2.4(0.5,4.3)$ & 0.341 \\
\hline$<400 \mathrm{IU} / \mathrm{ml}$ & $4.3(0.1,9.6)$ & $2.8(2.4,3.2)$ & $0.022^{*}$ \\
\hline \multicolumn{4}{|l|}{ Age } \\
\hline$\geq 60$ & $2.6(0.1,7.0)$ & $2.4(1.8,3.0)$ & 0.079 \\
\hline$<60$ & $2.0(0.1,5.7)$ & $2.4(1.7,3.1)$ & 0.124 \\
\hline \multicolumn{4}{|l|}{ Extent of disease } \\
\hline Locally advanced & $2.2(0.9,3.5)$ & $2.4(1.7,3.1)$ & 0.203 \\
\hline Metastatic & $3.7(0.1,7.6)$ & $2.4(2.2,2.6)$ & 0.053 \\
\hline
\end{tabular}

Table 4. Summary of adverse events ${ }^{\mathrm{a}}$ associated with chemotherapy

\begin{tabular}{|c|c|c|c|c|}
\hline \multirow[t]{2}{*}{ Adverse events } & \multicolumn{2}{|c|}{ XELIRI $(n=30)$} & \multicolumn{2}{|l|}{$\mathrm{IRI}(n=30)$} \\
\hline & $\begin{array}{l}\text { Grade 3/ } \\
4, n(\%)\end{array}$ & $\begin{array}{l}\text { All grades, } \\
n(\%)\end{array}$ & $\begin{array}{l}\text { Grade 3/ } \\
4, n(\%)\end{array}$ & $\begin{array}{l}\text { All grades, } \\
n(\%)\end{array}$ \\
\hline \multicolumn{5}{|l|}{ Haematological } \\
\hline Leucopaenia & $8(26.7 \%)$ & $28(93.3 \%)$ & $8(26.7 \%)$ & $27(90.0 \%)$ \\
\hline Neutropaenia & $8(26.7 \%)$ & 25 (83.3\%) & $7(23.3 \%)$ & $26(86.7 \%)$ \\
\hline Thrombocytopaenia & $2(6.7 \%)$ & $13(43.3 \%)$ & 1 (3.3\%) & $15(50.0 \%)$ \\
\hline Anaemia & $2(6.7 \%)$ & $20(66.7 \%)$ & $3(10.0 \%)$ & $21(70.0 \%)$ \\
\hline \multicolumn{5}{|l|}{ Gastrointestinal } \\
\hline Diarrhoea & $1(3.3 \%)$ & $8(26.7 \%)$ & $1(3.3 \%)$ & 7 (23.3\%) \\
\hline Nausea & $3(10.0 \%)$ & $27(90.0 \%)$ & $2(6.7 \%)$ & $25(83.3 \%)$ \\
\hline Vomiting & $1(3.3 \%)$ & 10 (33.3\%) & $1(3.3 \%)$ & $12(40.0 \%)$ \\
\hline \multicolumn{5}{|l|}{ General and laboratory } \\
\hline Hand-foot syndrome & $2(6.7 \%)$ & $16(53.3 \%)$ & $0(0 \%)$ & $0(0 \%)$ \\
\hline Stomatitis & $0(0 \%)$ & $5(16.7 \%)$ & $0(0 \%)$ & $3(10.0 \%)$ \\
\hline $\begin{array}{l}\text { Liver enzyme } \\
\text { elevation }\end{array}$ & $0(0 \%)$ & $10(33.3 \%)$ & $0(0 \%)$ & $8(26.7 \%)$ \\
\hline
\end{tabular}

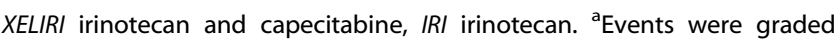
according to CTCAE v4.0

chemotherapy including docetaxel (8 patients) and S-1 (5 patients). In the IRI-arm, 11 patients (36.7\%) received post-study chemotherapy including capecitabine (4 patients), docetaxel (6 patients), and S-1 (1 patient).

\section{DISCUSSION}

The treatment beyond disease progression after GP doublet chemotherapy in ABTC patients remains a challenge. Until now, three independent systematic reviews have provided the most comprehensive results regarding the use of second-line chemotherapy in ABTC. ${ }^{19-21}$ However, the survival data reported in these studies were not satisfying.

Although there was no solid evidence that indicates any clear survival benefit of the use of second-line chemotherapy, irinotecan had been preliminary evaluated as a monotherapy or as part of combination therapies in ABTC, and the median OS for second-line treatment was approximately 6-8 months. ${ }^{15,16,22}$ Since all the patients recruited in this study preserved a good PS and require continuing care, it was reasonable and ethical to choose irinotecan monotherapy as control. However, whether there is a definite survival advantage of irinotecan-containing regimens over irinotecan monotherapy is still unclear. Capecitabine is another active agent which had been substantially studied for the anti-tumour effect in ABTC patients. ${ }^{11,23-25}$ More recently, capecitabine had established its fundamental role as the standard adjuvant therapy in BTC for a median OS of 53 months without significant impairment of quality of life (BILCAP study). ${ }^{17}$ Thus, XELIRI regimen was chosen as the study scheme.

On the basis of considerations above, we started this randomised, phase II study to evaluate the anti-tumour activity and safety of XELIRI regimen over irinotecan monotherapy in GP refractory ABTC patients. In the present study, although the increase in OS was not significant, XELIRI regimen prolonged the second-line PFS of ABTC patients by 1.3 months, increased the 9month survival rate by approximate $30 \%$ over irinotecan alone (all $P<0.05)$. The relatively high $\mathrm{RR}$ and two cases with $C R$ in the XELIRI-arm were also impressive. More importantly, the survival benefit in this study was achieved with the addition of an outpatient capecitabine schedule without an increase of hospitalisation. These data provided evidence that XELIRI regimen is an effective second-line treatment option for ABTC patients when compared to irinotecan monotherapy.

Although the BICC-C study found CapelRI regimen (irinotecan $250 \mathrm{mg} / \mathrm{m}^{2}$ on day 1 , capecitabine $1000 \mathrm{mg} / \mathrm{m}^{2}$ twice daily on days $1-14$, every 3 weeks) was more toxic that impeded its acceptance as an active regimen in metastatic colorectal cancer, ${ }^{26}$ the entire toxicity profile observed in this study was similar between the two treatment arms, only the incidence of hand-foot syndrome and stomatitis was increased in the XELIRI-arm, which was inevitable for the addition of capecitabine. We believe the dose adjustment of irinotecan and capecitabine from a 3-week schedule to a 2-week schedule may contribute to the better tolerance of this combination. Actually, several studies using a dose-adjusted irinotecan and capecitabine regimen had also revealed a better tolerance and efficacy in metastatic colorectal cancer. ${ }^{27-29}$ Therefore, optimising the dose intensity and administration scheme would be of great importance in maximising the efficacy of irinotecan and capecitabine combination, regardless of tumour origin.

There were several studies focusing on the selection of ABTC patients who might benefit from second-line chemotherapy. Parameters including better ECOG PS, lower serum CA 19-9 level, longer PFS after first-line chemotherapy, and less metastatic organs are considered better prognostic factors for ABTC in this setting. ${ }^{30,31}$ In this study, we also studied the prognostic factors including primary site, CA 19-9 level, age, and extent of disease, as shown in Table 3; patients with higher serum CA 19-9 level showed worse PFS than those with lower serum CA 19-9 level, this being consistent with previous reports. ${ }^{31}$ It is also important to note that the XELIRI regimen showed longer PFS in patients with serum CA $19-9$ level $<400 \mathrm{lU} / \mathrm{ml}$ and non-gallbladder origin, suggesting these two subgroup patients are more likely to benefit from the XELIRI combination therapy.

There were also several limitations to this study. First, as BTC is still a rather rare cancer, and the study was planned and conducted at a single centre, the sample size in this study was relatively small, which could have selection bias. Second, more extrahepatic cholangiocarcinoma patients with jaundice and extensive disease patients with poor PS were excluded according to the eligibility criteria; thus, the proportions of intrahepatic cholangiocarcinoma and locally advanced disease patients were relatively higher in this study. Third, according to recent studies, single agent capecitabine may served as a better second-line 
control arm, but at the time of our study design, the results of BILCAP study was far before reported. Therefore, according to the institute's experience and literature review, irinotecan was chosen as control arm. A phase III study of larger data sets with multicentre participation is warranted in the future.

In conclusion, this is the first randomised, phase II study to evaluate the efficacy and safety of irinotecan-containing regimens in good PS second-line ABTC patients. This study met its primary objective, and the outcomes from this study showed a clear advantage of XELIRI regimen over irinotecan monotherapy in prolonging PFS, with acceptable toxicity. XELIRI regimen merits further evaluation in largepopulation, multi-centre phase III trials in ABTC.

\section{ACKNOWLEDGEMENTS}

This work was supported by grants from National Natural Science Foundation of China (81472346)

\section{AUTHOR CONTRIBUTIONS}

W.W. and W.F. designed the study, guided data analysis and interpretation and revised the manuscript critically for important intellectual content. Y.Z., X.T., P.Z., W.J., L.L., Z.T. and H.Z. conducted the clinical trial. Y.Z. and X.T. collected clinical and pathological information from the cancer patients, analysed data and wrote the manuscript. All authors reviewed the manuscript.

\section{ADDITIONAL INFORMATION}

Competing interests: The authors declare no competing interests.

Availability of data and materials: All data are fully available without restriction.

Note: This work is published under the standard license to publish agreement. After 12 months the work will become freely available and the license terms will switch to a Creative Commons Attribution 4.0 International (CC BY 4.0).

\section{REFERENCES}

1. Siegel, R. L., Miller, K. D. \& Jemal, A. Cancer statistics, 2018. CA A Cancer J. Clin. 68 7-30 (2018).

2. Razumilava, N. \& Gores, G. J. Cholangiocarcinoma. Lancet 383, 2168-2179 (2014).

3. Misra, S., Chaturvedi, A., Misra, N. C. \& Sharma, I. D. Carcinoma of the gallbladder. Lancet Oncol. 4, 167-176 (2003).

4. Okusaka, T. et al. Phase II study of single-agent gemcitabine in patients with advanced biliary tract cancer. Cancer Chemother. Pharmacol. 57, 647-653 (2006).

5. Yonemoto, N. et al. A multi-centre retrospective analysis of survival benefits of chemotherapy for unresectable biliary tract cancer. Jpn. J. Clin. Oncol. 37, 843-851 (2007)

6. Valle, J. et al. Cisplatin plus gemcitabine versus gemcitabine for biliary tract cancer. N. Engl. J. Med. 362, 1273-1281 (2010).

7. Okusaka, T. et al. Gemcitabine alone or in combination with cisplatin in patients with biliary tract cancer: a comparative multicentre study in Japan. Br. J. Cancer 103, 469-474 (2010)

8. Kim, M. J. et al. Gemcitabine-based versus fluoropyrimidine-based chemotherapy with or without platinum in unresectable biliary tract cancer: a retrospective study. BMC Cancer 8, 374 (2008).

9. Kim, B. J. et al. Efficacy of fluoropyrimidine-based chemotherapy in patients with advanced biliary tract cancer after failure of gemcitabine plus cisplatin: retrospective analysis of 321 patients. Br. J. Cancer 116, 561-567 (2017).

10. Kobayashi, S. et al. Phase II study of fixed dose-rate gemcitabine plus S-1 as a second-line treatment for advanced biliary tract cancer. Cancer Chemother. Pharmacol. 80, 1189-1196 (2017).
11. Jung, J. H. et al. Combination therapy with capecitabine and cisplatin as secondline chemotherapy for advanced biliary tract cancer. Chemotherapy 62, 361-366 (2017).

12. Kim, R. et al. Phase 2 study of combination SPI-1620 with docetaxel as second-line advanced biliary tract cancer treatment. Br. J. Cancer 117, 189-194 (2017).

13. Venturini, M. et al. Chemoembolization with drug eluting beads preloaded with irinotecan (DEBIRI) vs doxorubicin (DEBDOX) as a second line treatment for liver metastases from cholangiocarcinoma: a preliminary study. Br. J. Radiol. 89, 20160247 (2016).

14. Endlicher, E. et al. Irinotecan plus gemcitabine and fluorouracil in advanced biliary tract cancer: a retrospective study. Digestion 93, 229-233 (2016).

15. Sasaki, T. et al. A pilot study of salvage irinotecan monotherapy for advanced biliary tract cancer. Anticancer Res. 33, 2619-2622 (2013).

16. Ramaswamy, A. et al. Second-line palliative chemotherapy in advanced gall bladder cancer, CAP-IRI: safe and effective option. J. Gastrointest. Cancer 47, 305-312 (2016).

17. Primrose J. N. F. R., Palmer D. H. Adjuvant capecitabine for biliary tract cancer: The BILCAP Randomized Study. ASCO Annual Meeting 2017. Abstract 4006.

18. Simon, R., Wittes, R. E. \& Ellenberg, S. S. Randomized phase II clinical trials. Cancer Treat. Rep. 69, 1375-1381 (1985).

19. Brieau, B. et al. Second-line chemotherapy for advanced biliary tract cancer after failure of the gemcitabine-platinum combination: a large multicentre study by the Association des Gastro-Enterologues Oncologues. Cancer 121, 3290-3297 (2015).

20. Fornaro, L. et al. Second-line chemotherapy in advanced biliary cancer progressed to first-line platinum-gemcitabine combination: a multicentre survey and pooled analysis with published data. J. Exp. Clin. Cancer Res. 34, 156 (2015).

21. Lamarca, A., Hubner, R. A., David Ryder, W. \& Valle, J. W. Second-line chemotherapy in advanced biliary cancer: a systematic review. Ann. Oncol. 25 2328-2338 (2014).

22. Larsen, F. O., Markussen, A., Diness, L. V. \& Nielsen, D. Efficacy and safety of capecitabine, irinotecan, gemcitabine, and bevacizumab as second-line treatment in advanced biliary tract cancer: a phase II study. Oncology 94, 19-24 (2017).

23. Woo, S. M. et al. Gemcitabine plus cisplatin versus capecitabine plus cisplatin as first-line chemotherapy for advanced biliary tract cancer: a retrospective cohort study. Chemotherapy 59, 232-238 (2013).

24. Lee, J., Hong, T. H., Lee, I. S., You, Y. K. \& Lee, M. A. Comparison of the efficacy between gemcitabine-cisplatin and capecitabine-cisplatin combination chemotherapy for advanced biliary tract. Cancer Cancer Res. Treat. 47, 259-265 (2015).

25. Riechelmann, R. P., Townsley, C. A., Chin, S. N., Pond, G. R. \& Knox, J. J. Expanded phase II trial of gemcitabine and capecitabine for advanced biliary cancer. Cancer 110, 1307-1312 (2007)

26. Fuchs, C. S. et al. Randomized, controlled trial of irinotecan plus infusional, bolus, or oral fluoropyrimidines in first-line treatment of metastatic colorectal cancer: results from the BICC-C Study. J. Clin. Oncol. 25, 4779-4786 (2007).

27. Schmiegel, W. et al. Capecitabine/irinotecan or capecitabine/oxaliplatin in combination with bevacizumab is effective and safe as first-line therapy for metastatic colorectal cancer: a randomized phase II study of the AIO colorectal study group. Ann. Oncol. 24, 1580-1587 (2013).

28. Kotaka, M. et al. Study protocol of the Asian XELIRI ProjecT (AXEPT): a multinational, randomized, non-inferiority, phase III trial of second-line chemotherapy for metastatic colorectal cancer, comparing the efficacy and safety of XELIRI with or without bevacizumab versus FOLFIRI with or without bevacizumab. Chin. J. Cancer 35, 102 (2016).

29. Hamamoto, Y. et al. A phase I/II study of XELIRI plus bevacizumab as second-line chemotherapy for Japanese patients with metastatic colorectal cancer (BIX study). Oncologist 19, 1131-1132 (2014).

30. Kang, E. J. et al. Prognostic factors for the selection of patients eligible for secondline chemotherapy in advanced biliary tract cancer. Chemotherapy 60, 91-98 (2014).

31. Fornaro, L. et al. Multivariate prognostic factors analysis for second-line chemotherapy in advanced biliary tract cancer. Br. J. Cancer 110, 2165-2169 (2014). 